\title{
"Scanning mutagenesis" of the amino acid sequences flanking phosphorylation site 1 of the mitochondrial pyruvate dehydrogenase complex
}

\author{
Nagib Ahsan ${ }^{1,2}$, Kirby N. Swatek ${ }^{1,2}$, Jingfen Zhang ${ }^{2,3}$, Ján A. Miernyk ${ }^{1,2,4 *}$, Dong $\mathrm{Xu}^{2,3}$ and \\ Jay J. Thelen ${ }^{1,2}$
}

${ }^{1}$ Department of Biochemistry, University of Missouri, Columbia, MO, USA

2 Interdisciplinary Plant Group, University of Missouri, Columbia, MO, USA

${ }^{3}$ Department of Computer Science, University of Missouri, Columbia, MO, USA

${ }^{4}$ Plant Genetics Research Unit, USDA, Agricultural Research Service, University of Missouri, Columbia, MO, USA

Edited by:

Steven Huber, US Department of Agriculture, USA

\section{Reviewed by:}

Jun Ito, Lawrence Berkeley National Laboratory, USA

Benjamin Schwessinger, University of California Davis, USA

\section{*Correspondence:}

Ján A. Miernyk, Plant Genetics

Research Unit, USDA, Agricultural

Research Service, 102 Curtis Hall,

University of Missouri, Columbia,

MO 65211, USA.

e-mail: jan.miernyk@ars.usda.gov
The mitochondrial pyruvate dehydrogenase complex (mtPDC) is regulated by reversible seryl-phosphorylation of the E1a subunit by a dedicated, intrinsic kinase. The phospho-complex is reactivated when dephosphorylated by an intrinsic PP2C-type protein phosphatase. Both the position of the phosphorylated Ser-residue and the sequences of the flanking amino acids are highly conserved. We have used the synthetic peptide-based kinase client $(\mathrm{KiC})$ assay plus recombinant pyruvate dehydrogenase $\mathrm{E} 1 \alpha$ and E1 $\alpha$-kinase to perform "scanning mutagenesis" of the residues flanking the site of phosphorylation. Consistent with the results from "phylogenetic analysis" of the flanking sequences, the direct peptide-based kinase assays tolerated very few changes. Even conservative changes such as Leu, Ile, or Val for Met, or Glu for Asp, gave very marked reductions in phosphorylation. Overall the results indicate that regulation of the mtPDC by reversible phosphorylation is an extreme example of multiple, interdependent instances of co-evolution.

\section{Keywords: KiC assay, mass specrometry, mitochondrial, phosphorylation site, pyruvate dehydrogenase complex,} synthetic peptides

\section{INTRODUCTION}

The mitochondrial pyruvate dehydrogenase complex (mtPDC) is a $10 \mathrm{MDa}$ nanomachine consisting of multiple copies of three central enzymes (Mooney et al., 2002). Pyruvate dehydrogenase (E1) catalyzes the oxidative decarboxylation of pyruvate using thiamine-pyrophosphate as a cofactor, followed by the reductive-acetylation of lipoyl moieties covalently linked to dihydrolipoamide acetyltransferase (E2) which transfers the acetyl-group to $\mathrm{CoASH}$, releasing acetyl-CoA as a product. Dihydrolipoyl dehydrogenase (E3) contains a FAD cofactor, and completes the reaction cycle by reoxidizing the lipoyl groups of $\mathrm{E} 2$, using $\mathrm{NAD}^{+}$as the electron acceptor.

The core of mtPDC is a pentagonal dodecahedron consisting of $60 \mathrm{E} 2$ polypeptides arranged with icosahedral symmetry (Reed, 1974; Thelen et al., 1999; Smolle et al., 2006). The E1 and E3 enzymes are non-covalently associated with the E2 core structures. The quaternary structure of mtPDC E1 is an $\alpha 2 \beta 2-$ heterotetramer (Fries et al., 2003). Overall, mtPDCs are very large structures with diameters of up to $50 \mathrm{~nm}$. They can be easily visualized in vitro by either negative staining transmission electron microscopy (Stoops et al., 1992) or cryo-electron microscopy (Milne et al., 2006). The complex molecular architecture allows enhanced catalytic efficiency, achieved by a combination of active-site coupling and substrate channeling (Perham, 2000; Perham et al., 2002).
The mtPDC occupies a strategic and overlapping position in catabolic and anabolic metabolism, and activity is regulated by multiple posttranslational mechanisms including product and metabolite inhibition, and reversible multi-site phosphorylation catalyzed by intrinsic kinase (PDK) and phosphatase (PDP) components (Mooney et al., 2002; Tovar-Méndez et al., 2003). Phosphorylation of the E1 $\alpha$ subunit by PDK inactivates the PDC, which is then reactivated by PDP-catalyzed dephosphorylation. The relative position of the Ser residue that comprises regulatory phosphorylation site 1 (Yeaman et al., 1978) plus the immediate flanking residues are conserved in all mtPDC sequences. Sites 2 and 3 have been defined only in mammalian sequences, and it is believed that Saccharomyces cerevisiae is phosphorylated only at site 1 (Gruhler et al., 2005; Krause-Buchholz et al., 2006).

There have been a very limited number of studies aimed at characterizing the effects of changes to amino acids flanking site 1 (Sugden et al., 1979; Mullinax et al., 1985; Hirani et al., 2011). Herein we report results from analysis of a series of systematic changes to Ser-flanking residues on phosphorylation of site 1 by $\mathrm{PDK}$, using the synthetic peptide-based kinase client $(\mathrm{KiC})$ assay (Huang et al., 2010).

\section{METHODS AND MATERIALS SYNTHETIC PEPTIDES}

A library of YHGHSMSDPGSTYR-variants consisting of 59 14-mer peptides was synthesized by the Sigma Chemical Co. 
(PEPscreen, St. Louis, MO, USA). The sequences are listed in Table A1. Stock solutions were prepared by dissolving the peptides in $80 \%(\mathrm{v} / \mathrm{v})$ dimethylformamide in $\mathrm{H}_{2} \mathrm{O}$ to a final concentration of $8 \mathrm{mM}$. Peptides (Table A1) were further diluted from the stock solutions into the assays.

\section{ASSAY OF PDC E1 $\alpha$-KINASE ACTIVITY}

Recombinant His6-AtPDK was prepared as described previously (Tovar-Méndez et al., 2005). The synthetic peptide-based KiC assay (Huang et al., 2010) was used with minor modifications. Assays were conducted for $1 \mathrm{~h}$ at $37^{\circ} \mathrm{C}$, with $500 \mathrm{rpm}$ agitation. The assay volume was $40 \mu \mathrm{L}$, and peptides were tested at a final concentration of $20 \mu \mathrm{M}$. After assay, samples were immediately frozen and freeze-dried.

Prior to MS analysis, freeze dried-samples were dissolved by adding $40 \mu \mathrm{L}$ of $0.1 \%$ formic acid. Half of each sample was added to a 96-well plate which was then placed onto a $10^{\circ} \mathrm{C}$ cooled auto-sampler. Ten microliter of each sample was analyzed using a Finnigan Surveyor liquid chromatography (LC) system coupled with an LTQ Orbitrap XL ETD mass spectrometer (Thermo Fisher, San Jose, CA). After injection, peptides were bound to a C8 Captrap (Michrom Bioresources, Auburn, CA, USA), eluted with a gradient of 1 to $30 \%$ acetonitrile (ACN) in $0.1 \%$ formic acid over a period of $15 \mathrm{~min}$, and then separated using a "Magic C18” (200 A, $5 \mu$ bead, Michrom Bioresources) fused silica column $(10 \mathrm{~cm} \times 150 \mu$, Polymicro Technologies, Phoenix, AZ). The C8 Captrap and fused silica column were purged with two spikes of $80 \%$ ACN over a period of $6 \mathrm{~min}$, before being re-equilibrated to $0.1 \%$ formic acid. Prior to MS analysis, the C18-fused silica column was pre-equilibrated with a gradient of $100-0 \%$ ACN in $0.1 \%$ formic acid to rid the matrix of any contaminating ions. Instrument settings were similar to those used previously (Huang et al., 2010). Precursor mass values were scanned with the analyzer set to FTMS: mass range, normal; resolution, 100,000; scan type, full; polarity, positive; data type, centroid; and a scan range of $300-2000 \mathrm{~m} / \mathrm{z}$. Nanospray ionization source settings were: spray voltage, $2.1 \mathrm{kV}$; capillary temp, $250^{\circ} \mathrm{C}$; capillary voltage, $36 \mathrm{~V}$, and tube lens, $90 \mathrm{~V}$. The four most abundant ions from the precursor scan were selected for data-dependent acquisition using the ion trap-analyzer, normal mass range, normal scan rate, centroid data type, and a minimum signal threshold of 500 counts. Collision induced dissociation activation settings included: isolation width, $2.0 \mathrm{~m} / \mathrm{z}$; normalized collision energy, 35; and activation time, $30 \mathrm{~ms}$. Dynamic exclusion was disabled. The multi-stage activation acquisition mode was enabled with neutral loss masses of $-24.5,-32.7,-49.0$, and -98.0 .

\section{BIOINFORMATIC ANALYSIS}

The MS data were exported, processed, and compared with a decoy database consisting of the random complement of the sequences comprising the peptide library, using the SEQUEST algorithm (Proteome Discoverer 1.0, Thermo Fisher). The search parameter settings have been previously described (Huang et al., 2010). Identifications were evaluated using the XCorr versus charge state function of Proteome Discoverer, and peptide identities with a score of $>13.01$ (a probability of 0.05 ) were accepted. The XCorr values for each charge state were set to default, and no decoy hits were allowed. Spectra that passed this filtering were exported to PhosSite (in-house software, available for download at digbio.missouri.edu/p3db/tools) to assign specific sites of phosphorylation. For final validation, each spectrum was inspected manually, and accepted only when the phosphopeptide had the highest XCorr value, site-determining fragment ions allowed unambiguous localization of the phosphorylation site, and all major ions in the spectrum were accounted for.

Data presented as heat-maps (Figures 2 and $\mathbf{3}$ ) are from spectral counting, and are means of three technical replicates. While we considered the possibility unlikely, it is conceivable that nonphosphorylated peptides would not ionize as well as those that are phosphorylated, that they might not "fly" as well, or might in some other way be difficult to compare. To allay any concerns, all the data for detection of both phosphorylated and non-phosphorylated peptides is presented in the Figures A1-A3.

\section{D MODELING OF NATIVE $A$. thaliana PDC E1 $\alpha$ AND THE PHOSPHORYLATION SITE-FLANKING VARIANTS}

MUFOLD is an efficient and accurate protein tertiary structure prediction platform that applies whole and partial template information along with computational techniques (Zhang et al., 2010). For the target, AtPDC E1 $\alpha$, MUFOLD was used first to predict the secondary structure and solvent accessibility and then to scan the PDB for target/template alignments. The best templates are 1NI4_A, 2OZL_A, and 3EXE_A from the 1.2.4.1 enzyme family and 1UMD_A and 2BFF_A from the 1.2.4.4 enzyme family. These templates cover almost $95 \%$ of the target residues. However, the first $32 \mathrm{~N}$-terminal target residues are either not covered by any template or the predicted secondary structures (solvent accessibilities) are not matched with the templates. A similar situation exists for some C-terminal target residues. Thus, MUFOLD was used to search and evaluate fragmental structures which fit the target termini in terms of sequence, secondary structure, solvent accessibility, and 3D structure. Finally, MUFOLD used the structural constraints from templates and fragments to build the target model. In order to predict the local structure changes that resulted from each flanking region residue substitution, e.g., M293L (peptide 11), the overall process was repeated to build a 3D model.

\section{RESULTS AND DISCUSSION}

The E1 component of Type II PDC is an $\alpha 2 \beta 2$ heterotetramer. Mammalian mtPDC is regulated by multi-site phosphorylation of the E1 $\alpha$ subunit, catalyzed by the intrinsic PDK. While site 1 is preferentially phosphorylated by PDK, saturation phosphorylation of any one of the three sites on any single E1 $\alpha$ subunit completely inhibits PDC activity (Sugden and Randle, 1978). The PDK is docked on the inner-lipoyl domain of the dilipoyl-E2 subunits, and the flexibility of this domain allows a single kinase to phosphorylate more than one E1 $\alpha$ subunit (Roche et al., 2003). It is believed that phosphorylation at Ser 292 prevents thiamine-PP-induced ordering of the sequence loop carrying the phosphorylation site. The disordering of this phosphorylation-loop is caused by a steric clash between the phosphoryl-group at site 1 and the nearby Ser 294 , which nullifies a hydrogen-bonding network essential for maintaining the 
loop conformation (Kato et al., 2008). The disordered phosphorylation loop impedes binding of lipoyl-domains of the PDC E2 core to E1, preventing the reductive acetylation step. This results in disruption of substrate channeling, and leads to inactivation.

The first site 1 flanking sequence was experimentally determined by manual Edman sequencing of a tryptic peptide derived from bovine PDC previously incubated with $\gamma$ - ${ }^{32} \mathrm{P}$-ATP (Yeaman et al., 1978; Figures 4A,B). We have chosen to refer to site 1 as "Ser 292 ," based upon the sequence of Arabidopsis thaliana E1 $\alpha$ (Luethy et al., 1995). During the genome-sequencing era it has been established that the position of $\operatorname{Ser}_{292}$ and the sequences of the flanking amino acids are highly conserved in mtPDC E1 $\alpha$ sequences. When directly studied, it has been verified that $\operatorname{Ser}_{292}$ was phosphorylated by the intrinsic PDK (e.g., Bykova et al., 2003; Hirani et al., 2011).

Based upon our phylogenic comparisons, the sequences $\mathrm{Thr}_{285}$ to $\mathrm{Tyr}_{288}$, Gly290 to Gly297, and $\mathrm{Tyr}_{300}$ to $\mathrm{Arg}_{301}$ are invariant, suggesting functional necessity in terms of E1 catalysis, PDK docking or orientation, or possibly some aspect of phosphotransfer. Residue 283 is typically large and hydrophobic, although Thr or Ala is present in some invertebrate sequences (Figure 1). Residue 284 appears to be unconstrained (Asp, Glu, Val, Ile, Leu,

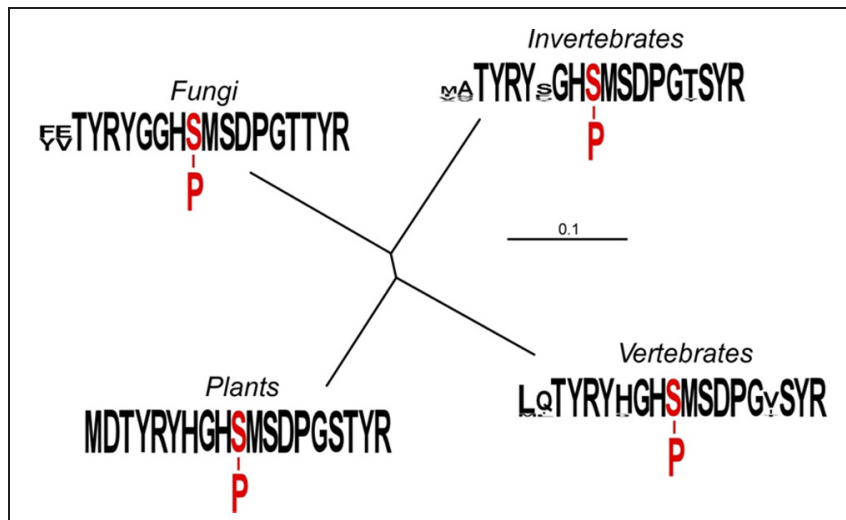

FIGURE 1 | Relationships among the amino acid sequences immediately flanking phosphorylation site $\mathbf{1}$ of mitochondrial PDC

E1 $\alpha$. The un-rooted tree was generated with TreeView version 1.6.6 (http://taxonomy.zoology.gla.ac.uk/rod/treeview.html). Each clade comprises 10 randomly selected sequences. The plant clade includes sequences of the "tryptic peptide" from Pisum sativum, A. thaliana, Glycine max, Lycopersicon esculentum, Solanum tuberosum, Beta vulgaris, Medicago truncatula, Zea mays, Oryza sativa, and Triticum aestivum. The sequences are identical. The vertebrate clade includes sequences from Homo sapiens, Rattus norvegicus, Gallus gallus, Macaca fascicularis, Xenopus laevis, Danio rerio, Oryctolagus cuniculus, Ailuropoda melanoleuca, Heterocephalus glaber, and Capsaspora owczarzaki. The invertebrate clade includes sequences from both nematodes and insects; Ascaris suum,

Caenorhabditis elegans, Bombyx mori, Aedes aegypti, Drosophila melanogaster, Daphnia pulex, Tribolium castaneum, Solenopsis invicta, Amblyomma maculatum, and Batrachochytrium dendrobatidis. The fungal clade includes sequences from Saccharomyces cerevisiae,

Schizosaccharomyces pombe, Kluyveromyces lactis, Naumovozyma dairenensis, Clavispora lusitaniae, Meyerozyma guilliermondii, Schizophyllum commune, Fusarium oxysporum, Verticillium alboatrum, and Ajellomyces capsulatus. The clade sequence presentations were assembled using WebLogo (Crooks et al., 2004).
Gln, Asn, or Ala). Residue 289 is most often Gly or His, but can also be Ser, Thr, or Phe in the invertebrate sequences. Residue 298 is most often Ser or Thr, but can also be Val, Ile, or Tyr. Residue 299 is Ser in both vertebrate and invertebrate sequences, but Thr in plants and fungi. The $\operatorname{Ser}_{299}$ residue is regulatory phosphorylation site 2 in mammalian PDC (Davis et al., 1977; Sugden et al., 1979; Mullinax et al., 1985).

Our "scanning mutagenesis" strategy, using synthetic peptides, recombinant PDK, and the quantitative $\mathrm{KiC}$ assay, yielded a set of results that would have been at least difficult to obtain using sitedirected mutagenesis of the intact protein sequence because of the occurrence of competing effects upon catalytic activity (e.g., Hirani et al., 2011). As suggested by the results from comparative sequence analyses, the site 1 flanking sequences tolerate little change. Only a few conserved "mutations" at specific positions allowed fractional activity (Figure 2, and Huang et al., 2010). In no instance did any peptide yield a hyperactive result. While the Y288A (peptide 58) is a drastic change, it was unexpected that a change three residues distal to $S_{292}$ would have such a large effect upon phosphorylation (Figure 2). Especially in light of the observation that the H289A (peptide 54) change had a much smaller inhibition. "Conservative" substitutions for His 289 (Lys, Arg, Asn; peptides 36-38, respectively) all resulted in a 55\% inhibition of phosphorylation of $\operatorname{Ser}_{292}$ (Figure 2). Changes of Gly290 to Ala (peptide 33) or Ser (peptide 34) inhibited phosphorylation of Ser 292 much less than did changes to Pro (peptide 32) or Thr

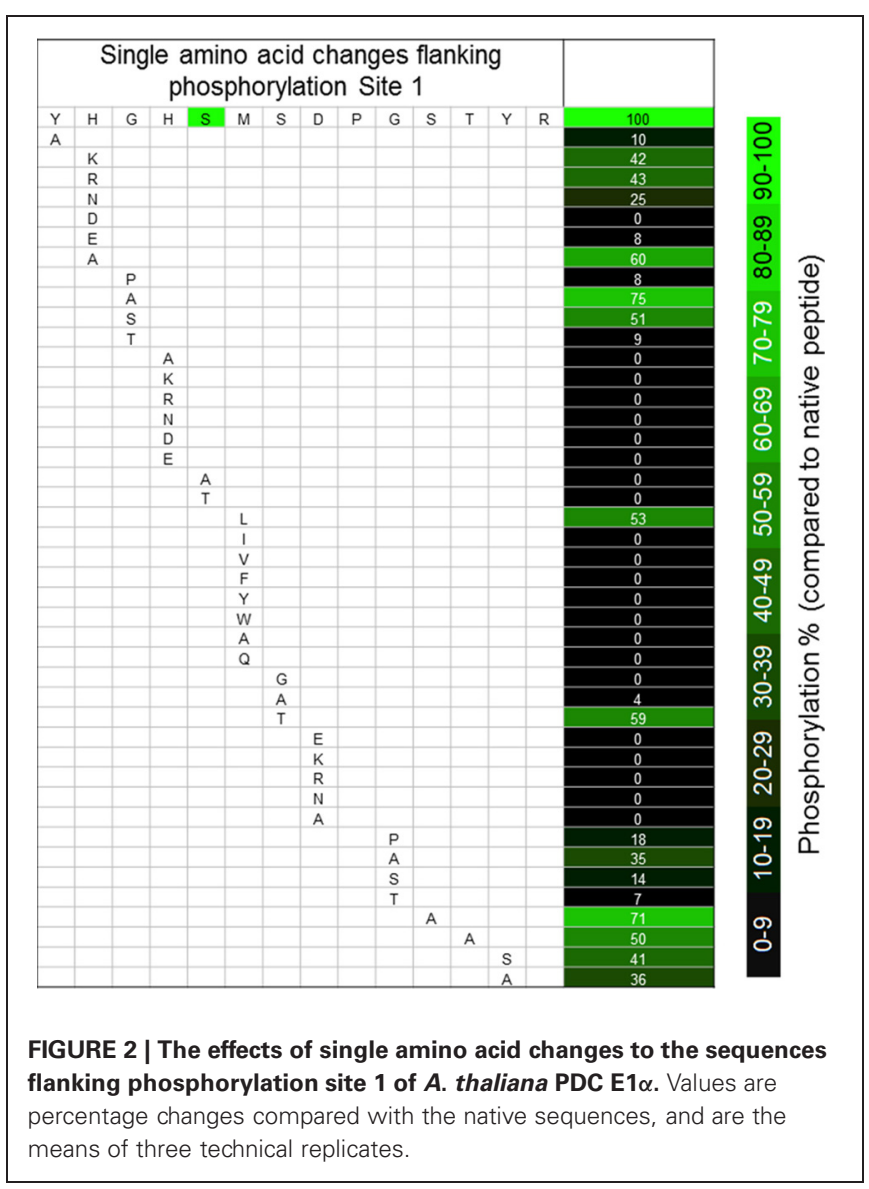


(peptide 35). Most changes to His 291 (peptides 3-5, 17, 18, 27-31) completely inhibited phosphorylation, and the S292A (peptide 48) and S292T (59) peptides were not phosphorylated (Figure 2). It has previously been reported that S292T peptides were not phosphoryl acceptors (Miernyk and Randall, 1989; Huang et al., 2010).

We have chosen to present our peptide phosphorylation data as spectral counts, a relatively simple format that is being increasingly used in proteomic analyses (Mueller et al., 2008; Huang et al., 2010; Thelen and Miernyk, 2012). While our numbers are not large, they are reproducible (Figures A1-A3), and it is clear that there are not any problems detecting and quantifying either phosphorylated or non-phosphorylated peptides.

Phosphorylation of the M293L (11) peptide was inhibited $50 \%$, but any other changes, conservative or otherwise, completely prevented phosphorylation of $\operatorname{Ser}_{292}$ (peptides 12-22; Figure 2, and Huang et al., 2010). The structural bases for the differences must be subtle because there do not appear to be any major perturbations (Figures 4D,E). It was previously reported that oxidation of $\mathrm{Met}_{293}$ to MetSO almost completely blocked phosphorylation of Ser $_{292}$ (Miernyk et al., 2009; Huang et al., 2010), and it was proposed that this reversible change might be of regulatory importance.

The S294T substitution (peptide 60) decreased phosphorylation of $\operatorname{Ser}_{292} 50 \%$, while changes to Ala (peptide 49) or Gly (peptide 46) inhibited it completely (Figure 2, and Huang et al., 2010). It has been proposed that Ser $_{294}$ might be involved in thiamin-PP binding (Kato et al., 2008). Our results suggest that it is the hydroxyl-group that is necessary for phosphorylation rather than simply spacing due to amino acid size ( $/$ /T v. A).

We observed that any changes to $\mathrm{Asp}_{295}$, including the conservative D295E (peptide 23) change, resulted in complete inhibition of $\operatorname{Ser}_{292}$ phosphorylation (Figure 2). From the predicted

\begin{tabular}{|c|c|c|c|c|c|c|c|c|c|c|c|c|c|c|}
\hline \multicolumn{14}{|c|}{$\begin{array}{l}\text { Multiple amino acid changes flanking } \\
\text { Phosphorylation Site } 1\end{array}$} & \multirow{2}{*}{100} \\
\hline \multirow[t]{17}{*}{ Y } & $\mathrm{H}$ & G & $\mathrm{H}$ & S & $M$ & $S$ & $D$ & $P$ & $G$ & $S$ & $\mathrm{~T}$ & Y & $R$ & \\
\hline & & & & & & & & & & $\mathrm{T}$ & $S$ & & & 93 \\
\hline & & & $S$ & $\mathrm{H}$ & & & & & & & & & & 0 \\
\hline & & & & $M$ & $S$ & & & & & & & & & 0 \\
\hline & & & & & $S$ & $M$ & & & & & & & & 0 \\
\hline & & & & & & & & G & $P$ & & & & & 0 \\
\hline & $S$ & $\mathrm{H}$ & $G$ & $\mathrm{H}$ & & & & & & & & & & 0 \\
\hline & & S & $\mathrm{G}$ & $\mathrm{H}$ & & & & & & & & & & 0 \\
\hline & & $M$ & G & $\mathrm{H}$ & $S$ & & & & & & & & & 0 \\
\hline & & & $M$ & $\mathrm{H}$ & $S$ & & & & & & & & & 0 \\
\hline & & & $\mathrm{M}$ & & D & & & & & & & & & 0 \\
\hline & & & $M$ & & $D$ & $P$ & $S$ & & & & & & & 0 \\
\hline & & & $\mathrm{M}$ & & D & $P$ & G & $S$ & & & & & & 0 \\
\hline & & & $\mathrm{M}$ & & $D$ & $P$ & $\mathrm{G}$ & S & $\mathrm{T}$ & & & & & 0 \\
\hline & & & & & $\mathrm{D}$ & $M$ & & & & & & & & 0 \\
\hline & & & & & $D$ & $P$ & $M$ & & & & & & & 0 \\
\hline & & & & & $D$ & $P$ & G & M & & & & & & 0 \\
\hline
\end{tabular}

FIGURE 3 | The effects of multiple simultaneous amino acid changes to the sequences flanking phosphorylation site 1 of $A$. thaliana PDC E1 $\alpha$. Values are percentage changes compared with the native sequences, and are the means of three technical replicates. structures, it can be seen that the D295K (Figure 4F) and D295E (Figure 4G) changes cause significant changes in the positioning/presentation of $\mathrm{Ser}_{294}$, Met 293 , and $\mathrm{Ser}_{292}$. Previous studies of the D295N change using peptide substrates with the mammalian PDK showed substantially reduced phosphorylation of $\mathrm{S}_{292}$, and it was proposed that Asp $_{295}$ is involved with PDK binding (Mullinax et al., 1985). The results of Mullinax et al. (1985) were recently verified by Hirani et al. (2011) using recombinant A. thaliana PDC-E1 and PDK. The D295N, D295A, and D295L site-directed mutants all had greatly reduced phosphorylation of $\mathrm{E} 1 \alpha$.

Because of an unfortunate oversight in our experimental design, we failed to systematically evaluate changes to $\mathrm{Pro}_{296}$. We believe, however, that the absolute conservation of Pro at this
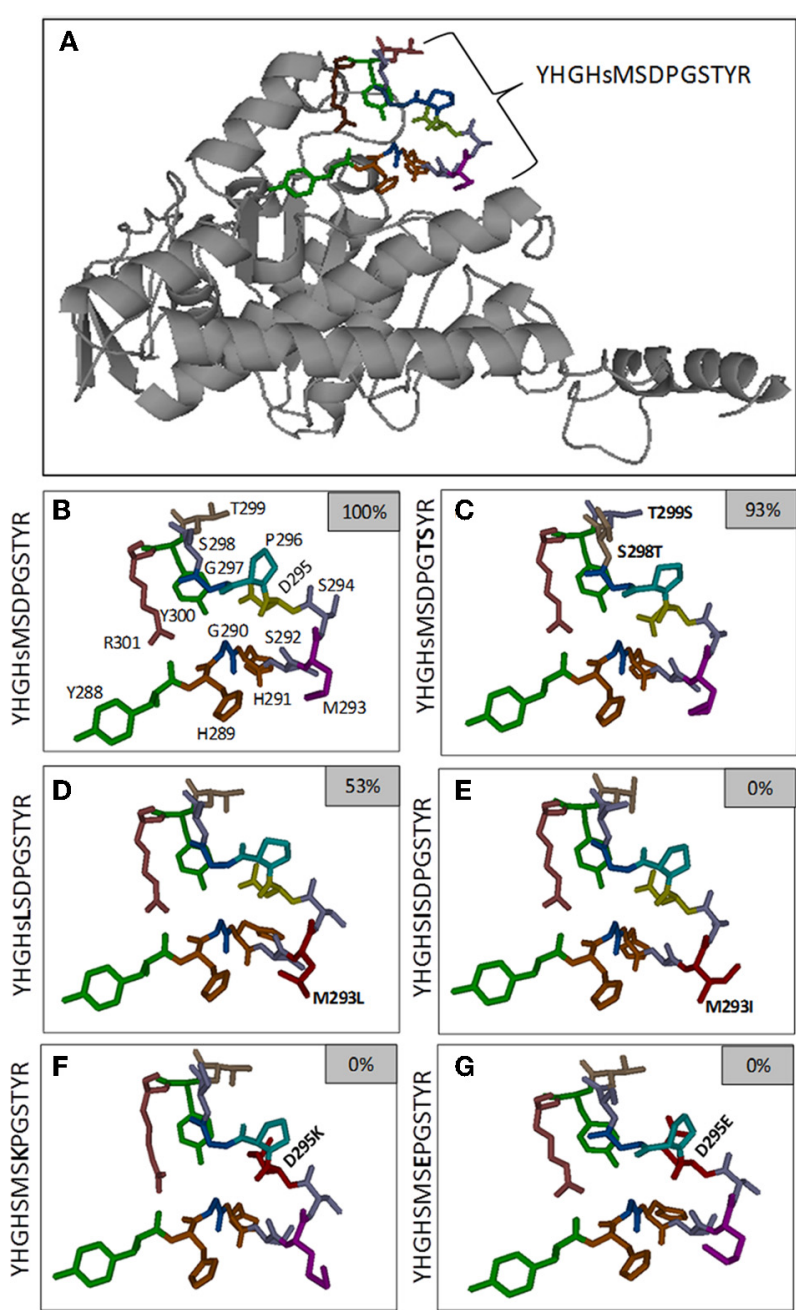

FIGURE 4 | Predicted local structure of the $A$. thaliana PDC E1 $\alpha$ subunit, with emphasis on the phosphorylation site $1\left(\operatorname{Ser}_{292}\right)$-flanking residues. Panel $(\mathbf{A})$ includes the $3 \mathrm{D}$ representation of the full-length $\mathrm{E} 1 \alpha$ subunit. Regulatory phosphorylation site 1 is shown as a colored stick-diagram. In panel (B), an enlarged version of the sequence flanking site 1 is presented. In panels $(\mathbf{C}-\mathbf{G})$ the stick-diagrams of selected amino acid substitution variants are presented. The small inset boxes indicate the extent of phosphorylation relative to the native sequence. 
position in all E1 $\alpha$ sequences (Figure 1), and the complete loss of phosphorylation resulting from the exchange of $\mathrm{PrO}_{296} \mathrm{Gly}_{297}$ for $\mathrm{Gly}_{296} \mathrm{Pro}_{297}$ (Figure 3) support our contention that any change from Pro at position 296 will also result in the loss of phosphorylation.

When residue 297 was changed from Gly to Pro (peptide 41), Ala (peptide 43), Ser (peptide 44), or Thr (peptide 45), phosphorylation of $\operatorname{Ser}_{292}$ was reduced from 65 to 90\% (Figure 2). Hirani et al. (2011) reported that with the G297S and G297D site-directed mutants the phosphorylation of $\operatorname{Ser}_{292}$ was only inhibited approximately $30 \%$. The phylogenetic sequence alignments revealed an absolute conservation of Gly at position 297 (Figure 1). As was found with substitutions for the N-terminal $\mathrm{Tyr}_{288}$, changes to the distal C-terminal Ser $298, \mathrm{Thr}_{299}, \mathrm{Tyr}_{300}$, and $\mathrm{Arg}_{301}$ residues significantly inhibited phosphorylation of $\mathrm{Ser}_{292}$ (30-60\%).

While even a single conservative change to a flanking amino acid might substantially reduce phosphorylation of $\operatorname{Ser}_{292}$ (Figure 2), nearly all multiple-changes completely abolished phosphorylation (Figure 3 and Table A1). This included the simple exchanging or scrambling of neighboring residues. The multiple changes probably reflect a combination of reduced accessibility of Ser 292 to PDK plus impaired recognition and/or binding. The single exception to this among the peptides screened was the switching of $\operatorname{Ser}_{298} / \mathrm{Thr}_{299}$ (peptide 2) which was without substantial effect on phosphorylation of $\operatorname{Ser}_{292}$ (Figure 3). This exchange converts the plant flanking-sequence to that of mammalian E1 $\alpha$ and recreates mammalian site 2 (Figure 1). Despite this, Ser $_{299}$ was not phosphorylated in our assays. When the predicted structure of peptide 2 (Table A1), which contains the Ser 298 / $\mathrm{Thr}_{299}$ to $\mathrm{Thr}_{298} /$ Ser $_{299}$ exchange, is examined (Figure 4C) it can be seen that in addition to other minor changes there is a significant change in the presentation of $\mathrm{D}_{295}$. While this does not affect phosphorylation of Ser 292 , it might contribute to Ser 299 not being phosphorylated. It is additionally noteworthy that all previous reports of phosphorylation of mammalian site 2 have been

\section{REFERENCES}

Bykova, N. V., Stensballe, A., Egsgaard, H., Jensen, O. N., and Møller, I. M. (2003). Phosphorylation of formate dehydrogenase in potato tuber mitochondria. J. Biol. Chem. 278, 26021-26030.

Crooks, G. E., Hon, G., Chandonia, J. M., and Brenner, S. E. (2004). WebLogo: a sequence logo generator. Genome Res. 14, 1188-1190.

Davis, P. F., Pettit, F. H., and Reed, L. J. (1977). Peptides derived from pyruvate dehydrogenase as substrates for pyruvate dehydrogenase kinase and phosphatase. Biochem. Biophys. Res. Commun. 75, 541-549.

Fries, M., Jung, H. I., and Perham, R. N. (2003). Reaction mechanism of the heterotetrameric $(\alpha 2 \beta 2)$ Elcomponent of 2-oxo acid dehydrogenase multienzyme complexes. Biochemistry 42, 6996-7002.
Gruhler, A., Olsen, J. V., Mohammed, S., Mortensen, P., Faergeman, N. J., Mann, M., and Jensen, O. N. (2005). Quantitative phosphoproteomics applied to the yeast pheromone signaling pathway. Mol. Cell Proteomics 4, 310-327.

Hirani, T. A., Tovar-Méndez, A., Miernyk, J. A., and Randall, D. D. (2011). Asp295 stabilizes the activesite loop structure of pyruvate dehydrogenase, facilitating phosphorylation of Ser292 by pyruvate dehydrogenase-kinase. Enzyme Res. doi: 10.4061/2011/939068. [Epub ahead of print].

Huang, Y., Houston, N. L., TovarMéndez, A., Stevenson, S. E., Miernyk, J. A., Randall, D. D., and Thelen, J. J. (2010). A quantitative mass spectrometry-based approach for identifying protein kinase clients and quantifying

done with either intact PDC or the E2-containing subcomplex present in the assays along with E1 and PDK.

\section{CONCLUSIONS}

Understanding the structure-function relationship between protein kinases and their clients is integral to understanding kinase specificity. Herein we demonstrate a facile approach for comprehensively and quantitatively assessing kinase specificity, using the MS-based KiC assay. There are two potential concerns with the use of MS for studying peptide-protein interactions; accurate discrimination of isobaric peptides, and differential ionization efficiency. To control for these two problems, respectively, we performed all peptide assays independently, and expressed phosphorylation efficiency as a ratio to the non-phosphorylated species. This assumes that phosphorylated peptides ionize with similar efficiency, which might otherwise be a concern in a more dramatic mutagenic schema.

The primary sequence drives formation of the secondary structure that underlies catalysis. In the case of the peptides being studied, there are primary sequence constraints relative to binding of both the substrate pyruvate and the co-factor thiamin-PP. At the same time, the evolutionary acquisition of reversible phosphorylation as a regulatory mechanism must have included the selection of primary sequence requirements necessary for kinase (and phosphatase) recognition and binding. It is the combination of these two interacting selective considerations, along with those related to the catalytic function of E1, that likely comprise the basis for the stringent primary-sequence specificity including and flanking "Ser 292 ."

\section{ACKNOWLEDGMENTS}

Research in Jay J. Thelen's lab is supported by the NSF and ILSIHealth Environmental Science Institute, and in Jan A. Miernyk's lab by the USDA, Agricultural Research Service, NSF, and the Nichols Foundation. M. L. Johnston prepared Figures 1-3.

kinase activity. Anal. Biochem. 402, 69-76.

Kato, M., Wynn, R. M., Chuang, J. L., Tso, S. C., Machius, M. Li, J., and Chuang, D. T. (2008). Structural basis for inactivation of the human pyruvate dehydrogenase complex by phosphorylation: role of disordered phosphorylation loops. Structure 16, 1849-1859.

Krause-Buchholz, U., Gey, U. Wünschmann, J., Becker, S. and Rödel, G. (2006). YIL042c and YOR090c encode the kinase and phosphatase of the Saccharomyces cerevisiae pyruvate dehydrogenase complex. FEBS Lett. 580, 2553-2560.

Luethy, M. H., Miernyk, J. A., and Randall, D. D. (1995). The mitochondrial pyruvate dehydrogenase complex: nucleotide and deduced amino-acid sequences of a cDNA encoding the Arabidopsis thaliana E1 $\alpha$-subunit. Gene 164, 251-254.

Miernyk, J. A., Johnston, M. L., Huber, S. C., Tovar-Méndez, A., Hoyos, E., and Randall, D. D. (2009). Oxidation of an adjacent methionine residue inhibits regulatory serylphosphorylation of pyruvate dehydrogenase. Proteomics Insights 2, 15-22.

Miernyk, J. A., and Randall, D. D. (1989). A synthetic peptidedirected antibody as a probe of the phosphorylation site of pyruvate dehydrogenase. J. Biol. Chem. 264, 9141-9144.

Milne, J. L., Wu, X., Borgnia, M. J., Lengyel, J. S., Brooks, B. R., Shi, D. Perham, R. N., and Subramaniam, S. (2006). Molecular structure of a 9-MDa icosahedral pyruvate dehydrogenase subcomplex containing the E2 and E3 enzymes using 
cryoelectron microscopy. J. Biol. Chem. 281, 4364-4370.

Mooney, B. P., Miernyk, J. A., and Randall, D. D. (2002). The complex fate of $\alpha$-ketoacids. Annu. Rev. Plant Biol. 53, 357-375.

Mueller, L. N., Brusniak, M. Y., Mani, D. R., and Aebersold, R. (2008). An assessment of software solutions for the analysis of mass spectrometry based quantitative proteomics data. J. Proteome Res. 7, 51-61.

Mullinax, T. R., Stepp, L. R., Brown, J. R., and Reed, L. J. (1985). Synthetic peptide substrates for mammalian pyruvate dehydrogenase kinase and pyruvate dehydrogenase phosphatase. Arch. Biochem. Biophys. 243, 655-659.

Perham, R. N. (2000). Swinging arms and swinging domains in multifunctional enzymes: catalytic machines for multistep reactions. Annu. Rev. Biochem. 69, 961-1004.

Perham, R. N., Jones, D. D., Chauhan, H. J., and Howard, M. J. (2002). Substrate channelling in 2-oxo acid dehydrogenase multienzyme complexes. Biochem. Soc. Trans. 30, $47-51$

Reed, L. J. (1974). Multienzyme complexes. Acc. Chem. Res. 7, 40-46.

Roche, T. E., Hiromasa, Y., Turkan, A., Gong, X., Peng, T., Yan, X., Kasten, S. A., Bao, H., and Dong,
J. (2003). Essential roles of lipoyl domains in the activated function and control of pyruvate dehydrogenase kinases and phosphatase isoform 1. Eur. J. Biochem. 270, 1050-1056.

Smolle, M., Prior, A. E., Brown, A. E. Cooper, A., Byron, O., and Lindsay, J. G. (2006). A new level of architectural complexity in the human pyruvate dehydrogenase complex. J. Biol. Chem. 281, 19772-19780.

Stoops, J. K., Baker, T. S., Schroeter J. P., Kolodziej, S. J., Niu, X.-D., and Reed, L. J. (1992). Threedimensional structure of the truncated core of the Saccharomyces cerevisiae pyruvate dehydrogenase complex determined from negative stain and cryoelectron microscopy images. J. Biol. Chem. 267, 14769-14775.

Sugden, P. H., Kerbey, A. L., Randle, P. J., Waller, C. A., and Reid, K. B. (1979). Amino acid sequences around the sites of phosphorylation in the pig heart pyruvate dehydrogenase complex. Biochem. J. 181, 419-426.

Sugden, P. H., and Randle, P. J. (1978). Regulation of pig heart pyruvate dehydrogenase by phosphorylation. Studies on the subunit and phosphorylation stoicheiometries. Biochem. J. 173, 659-668.
Thelen, J. J., and Miernyk, J. A. (2012) The proteomic future: where mass spectrometry should be taking us. Biochem. J. 444, 169-181.

Thelen, J. J., Muszynski, M. G., David, N. R., Luethy, M. H., Elthon, T. E., Miernyk, J. A., and Randall, D D. (1999). The dihydrolipoamide S-acetyltransferase subunit of the mitochondrial pyruvate dehydrogenase complex from maize contains a single lipoyl domain. J. Biol. Chem. 274, 21769-21775.

Tovar-Méndez, A., Hirani, T. A., Miernyk, J. A., and Randall, D. D. (2005). Analysis of the catalytic mechanism of pyruvate dehydrogenase kinase. Arch. Biochem. Biophys. 434, 159-168.

Tovar-Méndez, A., Miernyk, J. A., and Randall, D. D. (2003). Regulation of pyruvate dehydrogenase complex activity in plant cells. Eur. J. Biochem. 270, 1043-1049.

Yeaman, S. J., Hutcheson, E. T., Roche, T. E., Pettit, F. H., Brown, J. R., Reed, L. J., Watson, D. C., and Dixon, G. H. (1978). Sites of phosphorylation on pyruvate dehydrogenase from bovine kidney and heart. Biochemistry 17, 2364-2370

Zhang, J., Wang, Q., Barz, B., He, Z. Kosztin, I., Shang, Y., and $\mathrm{Xu}, \mathrm{D}$. (2010). MUFOLD: a new solution for protein $3 \mathrm{D}$ structure prediction. Proteins 78, 1137-1152.

Conflict of Interest Statement: The authors declare that the research was conducted in the absence of any commercial or financial relationships that could be construed as a potential conflict of interest.

Received: 15 February 2012; paper pending published: 07 March 2012; accepted: 19 June 2012; published online: 16 July 2012.

Citation: Ahsan N, Swatek KN, Zhang J, Miernyk JA, Xu D and Thelen JJ (2012)

"Scanning mutagenesis" of the amino acid sequences flanking phosphorylation site 1 of the mitochondrial pyruvate dehydrogenase complex. Front. Plant Sci. 3:153. doi: 10.3389/fpls.2012.00153

This article was submitted to Frontiers in Plant Proteomics, a specialty of Frontiers in Plant Science.

Copyright (c) 2012 Ahsan, Swatek, Zhang, Miernyk, Xu and Thelen. This is an open-access article distributed under the terms of the Creative Commons Attribution License, which permits use, distribution and reproduction in other forums, provided the original authors and source are credited and subject to any copyright notices concerning any thirdparty graphics etc. 


\section{APPENDIX}

Table A1 | Synthetic peptide-variants of the plant pyruvate dehydrogenase $E 1 \alpha$ phosphorylation site 1-flanking sequences.

\begin{tabular}{|c|c|c|}
\hline Number & Sequence & MW \\
\hline 1 & YHGHSMSDPGSTYR & 1594.6703 \\
\hline 2 & YHGHSMSDPGTSYR & 1594.6703 \\
\hline 3 & YHGSHMSDPGSTYR & 1594.6703 \\
\hline 4 & YHSGHMSDPGSTYR & 1594.6703 \\
\hline 5 & YSHGHMSDPGSTYR & 1594.6703 \\
\hline 6 & YHGHMSSDPGSTYR & 1594.6703 \\
\hline 7 & YHGHMSDSPGSTYR & 1594.6703 \\
\hline 8 & YHGHMSDPSGSTYR & 1594.6703 \\
\hline 9 & YHGHMSDPGSSTYR & 1594.6703 \\
\hline 10 & YHGHMSDPGSTSYR & 1594.6703 \\
\hline 11 & YHGHSLSDPGSTYR & 1576.7139 \\
\hline 12 & YHGHSISDPGSTYR & 1576.7139 \\
\hline 13 & YHGHSVSDPGSTYR & 1562.6982 \\
\hline 14 & YHGHSFSDPGSTYR & 1610.6982 \\
\hline 15 & YHGHSYSDPGSTYR & 1626.6931 \\
\hline 16 & YHGHSWSDPGSTYR & 1649.7091 \\
\hline 17 & YHGMHSSDPGSTYR & 1594.6703 \\
\hline 18 & YHMGHSSDPGSTYR & 1594.6703 \\
\hline 19 & YHGHSSMDPGSTYR & 1594.6703 \\
\hline 20 & YHGHSSDMPGSTYR & 1594.6703 \\
\hline 21 & YHGHSSDPMGSTYR & 1594.6703 \\
\hline 22 & YHGHSSDPGMSTYR & 1594.6703 \\
\hline 23 & YHGHSMSEPGSTYR & 1608.6859 \\
\hline 24 & YHGHSMSKPGSTYR & 1607.7383 \\
\hline 25 & YHGHSMSRPGSTYR & 1635.7445 \\
\hline 26 & YHGHSMSNPGSTYR & 1593.6863 \\
\hline 27 & YHGKSMSDPGSTYR & 1585.7063 \\
\hline 28 & YHGRSMSDPGSTYR & 1613.7125 \\
\hline 29 & YHGNSMSDPGSTYR & 1571.6543 \\
\hline 30 & YHGDSMSDPGSTYR & 1572.6383 \\
\hline 31 & YHGESMSDPGSTYR & 1586.6540 \\
\hline 32 & YHPHSMSDPGSTYR & 1634.7016 \\
\hline 33 & YHAHSMSDPGSTYR & 1608.6859 \\
\hline 34 & YHSHSMSDPGSTYR & 1624.6809 \\
\hline 35 & YHTHSMSDPGSTYR & 1638.6965 \\
\hline 36 & YKGHSMSDPGSTYR & 1585.7063 \\
\hline 37 & YRGHSMSDPGSTYR & 1613.7125 \\
\hline 38 & YNGHSMSDPGSTYR & 1571.6543 \\
\hline 39 & YDGHSMSDPGSTYR & 1572.6383 \\
\hline 40 & YEGHSMSDPGSTYR & 1586.6540 \\
\hline 41 & YHGHSMSDGPSTYR & 1594.6703 \\
\hline 42 & YHGHSMSDPPSTYR & 1634.7016 \\
\hline 43 & YHGHSMSDPASTYR & 1608.6859 \\
\hline 44 & YHGHSMSDPSSTYR & 1624.6809 \\
\hline
\end{tabular}

Table A1 | Continued.

\begin{tabular}{lll}
\hline Number & Sequence & MW \\
\hline 45 & YHGHSMSDPTSTYR & 1638.6965 \\
46 & YHGHSMGDPGSTYR & 1564.6597 \\
47 & YHGHSMSDPGSTSR & 1518.6390 \\
48 & YHGHAMSDPGSTYR & 1578.6754 \\
49 & YHGHSMADPGSTYR & 1578.6754 \\
50 & YHGHSASDPGSTYR & 1534.6669 \\
51 & YHGHSOSDPGSTYR & 1591.6884 \\
52 & YHGHSMSAPGSTYR & 1550.6805 \\
53 & YHGHSMSDPGATYR & 1578.6754 \\
54 & YAGHSMSDPGSTYR & 1528.6485 \\
55 & YHGASMSDPGSTYR & 1528.6485 \\
56 & YHGHSMSDPGSTAR & 1502.6441 \\
57 & YHGHSMSDPGSAYR & 1564.6597 \\
58 & AHGHSMSDPGSTYR & 1502.6441 \\
59 & YHGHTMSDPGSTYR & 1608.6859 \\
60 & YHGHSMTDPGSTYR & 1608.6859 \\
\hline
\end{tabular}




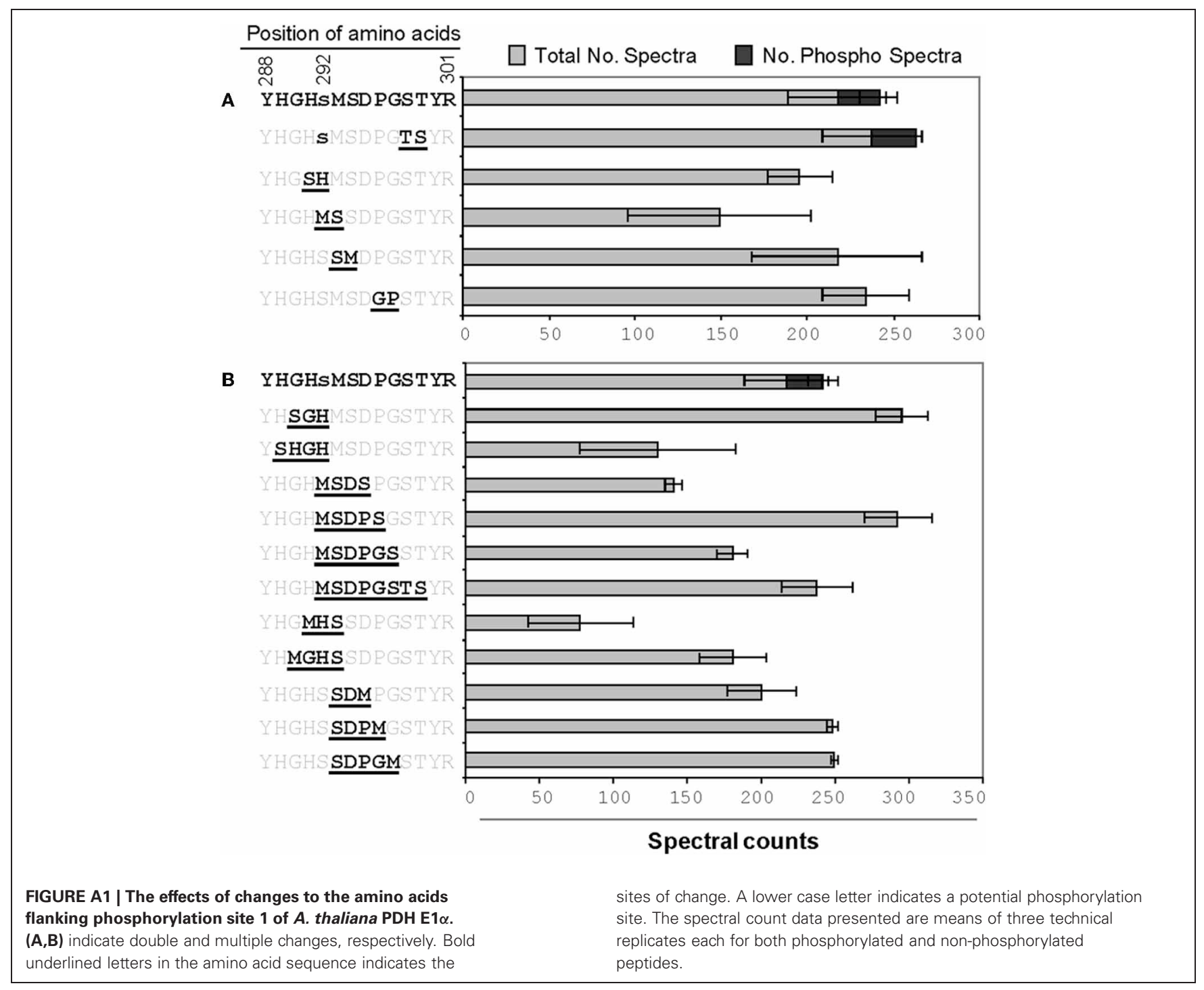


Position of amino acids

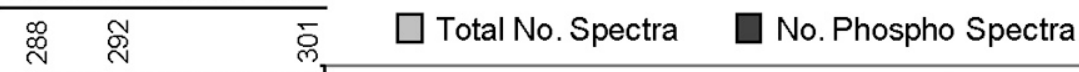

A YHGHsMSDPGSTYR

$\underline{\mathrm{K}} \mathbf{s}$

$\underline{\mathrm{R}} \mathbf{s}$

$\underline{\mathbf{N}} \mathbf{s}$

$\underline{\mathrm{D}}$

$\underline{\mathbf{E}} \mathbf{s}$

A $\mathbf{s}$

$\underline{A}$

$\underline{\mathrm{K}}$

$\underline{\mathbf{R}}$

N

D

$\underline{\mathbf{E}}$

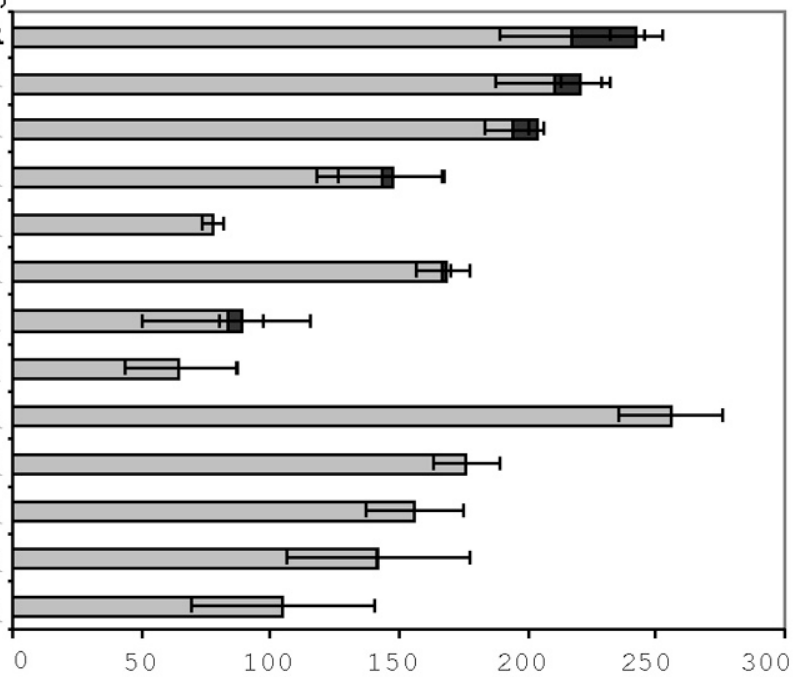

B YHGHSMSDPGSTYR
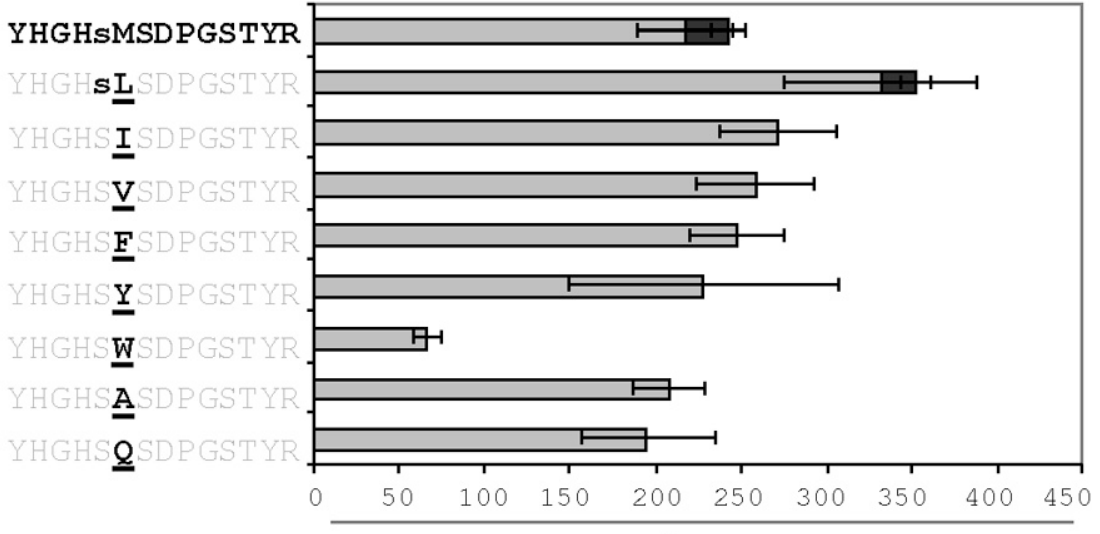

Spectral counts

FIGURE A2 | The effects of single changes to (A) $\mathbf{H i s}_{\mathbf{2 8 9}} / \mathbf{H i s}_{\mathbf{2 9 1}}$, or (B) $\mathbf{M e t}_{293}$. The spectral count data presented are means of three technical replicates each for both phosphorylated and non-phosphorylated peptides. 


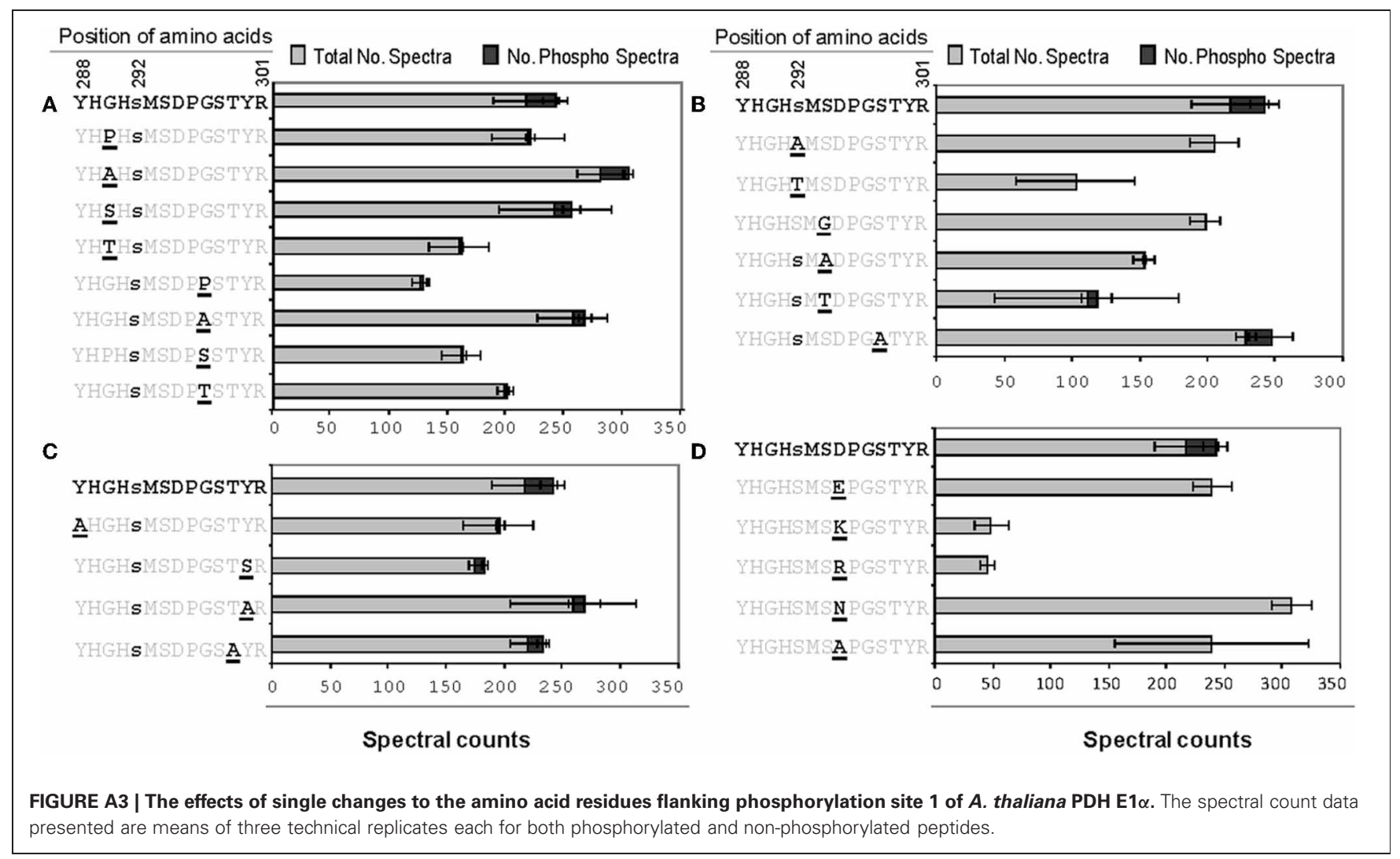

